\title{
Integrated analysis of dysregulated long non-coding RNAs/microRNAs/mRNAs in metastasis of lung adenocarcinoma
}

Lifeng $\mathrm{Li}^{1,3,5 \dagger}{ }^{\dagger}$, Mengle Peng ${ }^{2 \dagger}$, Wenhua Xue ${ }^{1 \dagger}$, Zhirui Fan ${ }^{3}$, Tian Wang ${ }^{3}$, Jingyao Lian ${ }^{3}$, Yunkai Zhai ${ }^{5}$, Wenping Lian', Dongchun Qin ${ }^{4^{*}}$ and Jie Zhao ${ }^{1,3,5^{*}}$ (1)

\begin{abstract}
Background: Lung adenocarcinoma (LUAD), largely remains a primary cause of cancer-related death worldwide. The molecular mechanisms in LUAD metastasis have not been completely uncovered.

Methods: In this study, we identified differentially expressed genes (DEGs), miRNAs (DEMs) and IncRNAs (DELs) underlying metastasis of LUAD from The Cancer Genome Atlas database. Intersection mRNAs were used to perform gene ontology (GO), Kyoto Encyclopedia of Genes and Genomes (KEGG) pathway and co-expression network analysis. In addition, survival analyses of intersection mRNAs were conducted. Finally, intersection mRNAs, miRNAs and IncRNAs were subjected to construct miRNA-mRNA-IncRNA network.

Results: A total of 1015 DEGs, 54 DEMs and 22 DELs were identified in LUAD metastasis and non-metastasis samples. GO and KEGG pathway analysis had proven that the functions of intersection mRNAs were closely related with many important processes in cancer pathogenesis. Among the co-expression interactions network, 22 genes in the coexpression network were over the degree 20. These genes imply that they have connections with many other gene nodes. In addition, 14 target genes (ARHGAP11A, ASPM, HELLS, PRC1, TMPO, ARHGAP30, CD52, IL16, IRF8, P2RY13, PRKCB, PTPRC, SASH3 and TRAF3IP3) were found to be associated with survival in patients with LUAD significantly (log-rank $P<0.05$ ). Two IncRNAs (LOC96610 and ADAM6) acting as ceRNAs were identified based on the miRNAmRNA-IncRNA network.
\end{abstract}

Conclusions: Taken together, the results may provide a novel perspective to develop a multiple gene diagnostic tool for LUAD prognosis, which might also provide potential biomarkers or therapeutic targets for LUAD.

Keywords: Lung adenocarcinoma, Metastasis, Biomarker, IncRNAs

\section{Background}

Lung cancer is the leading cause of cancer-related deaths worldwide, despite advances in lung cancer therapy, the average 5 -year survival rate is only $18 \%[1,2]$. A majority of the deaths associated with lung cancer are due to secondary disease or metastatic progression [3]. Lung

\footnotetext{
*Correspondence: qindongchun@163.com; jiezhaoz2016@163.com †Lifeng Li, Mengle Peng and Wenhua Xue contributed equally to this work ${ }^{1}$ Department of Pharmacy, The First Affiliated Hospital of Zhengzhou University, Zhengzhou 450052, Henan, China

${ }^{4}$ Department of Clinical Laboratory, The First Affiliated Hospital of Zhengzhou University, Key Laboratory of Laboratory Medicine of Henan Province, Zhengzhou 450052, Henan, China

Full list of author information is available at the end of the article
}

adenocarcinoma (LUAD) is a major lung cancer that is in a locally advanced or metastatic stage at the time of diagnosis, which leaves no time for early detection or treatment [4]. An early and accurate diagnosis may warrant timely treatment to potentially decrease the mortality. Therefore, molecular mechanisms that may help understand metastases of LUAD should be investigated to contribute to early diagnosis, better treatment and better overall prognosis of this disease.

Numerous published articles demonstrate that dysregulated genes are essential for initiation and progression of lung cancer. In recent years, the development of microarray technology has served as an effective measure to identify 
differentially expressed genes [5], and provides new insight into the alteration of gene expression during tumorigenesis [6]. Differentially expressed genes can be found through different experimental treatments, and their biological functions can be speculated via known information. The application of high-throughput miRNA profling methods, such as RNA sequencing and microarrays, has enabled researchers to identify a group of miRNAs as biomarkers in cancer diagnosis [7]. MicroRNAs (miRNAs) are a class of single stranded, non-coding RNAs of 19-25 nucleotides [8], which transcriptionally or post-transcriptionally regulate gene expression through binding to targeted mRNAs and influence the degradation and translation of mRNA [9]. Accumulating evidence suggests that aberrant levels of miRNAs are linked to proliferation, angiogenesis, and metastasis in various human malignancies [10]. Besides, miRNA has incurred broad attention as a targeting choice in cancer therapies [11] or as the diagnostic or prognostic markers [12]. Long non-coding RNAs (lncRNAs) are non-coding RNAs ranging in length from 200 nucleotides to $\sim 100 \mathrm{~kb}$ [13]. LncRNAs have mechanistically diverse functions in the cell, and in the nucleus, LncRNA has been shown to regulate gene expression in either cis or trans by recruiting a chromatin-modifying complex to the promoter of a target gene [14, 15]. Besides, it has been reported that lncRNAs are key competing endogenous RNAs (ceRNAs) harboring miRNA response elements (MREs) and serve as ceRNAs to exchange with mRNAs via competitively binding to common miRNAs [16].

The Cancer Genome Atlas (TCGA) is one prominent example of the renowned public databases which provides a platform of RNA sequencing with mRNA, miRNA and lncRNA data of various cancers. By integratively analysis RNA-Seq and miRNA-Seq data of LUAD samples from TCGA database, we successfully corhorted a set of differentially expression genes (DEGs), miRNAs (DEMs) and IncRNAs (DELs) underlying LUAD metastasis and non-metastasis samples. Based on intersection mRNAs, gene ontology (GO), Kyoto Encyclopedia of Genes and Genomes (KEGG) pathway enrichment and co-expression analysis were conducted. Furthermore, we performed a receiver operating characteristic (ROC) analysis to investigate the diagnostic value of intersection mRNAs. Finally, miRNA-mRNA-lncRNA network were constructed. Our study might provide a meaningful contribution to exploring the mechanisms of LUAD metastasis and candidate diagnostic biomarkers and therapeutic targets.

\section{Materials and methods}

\section{DEGs, DEMs and DELs of LUAD metastasis} and non-metastasis samples from TCGA data

The LUAD metastasis and non-metastasis RNA-Seq and miRNA-Seq data were downloaded from the TCGA database using The GDC Data Portal (https://gdc-porta l.nci.nih.gov/). The mRNA and lncRNA expression data included a total of 372 samples consisting of 25 LUAD metastasis and 347 non-metastasis samples. The miRNA expression data included a total of 305 samples consisting of 19 LUAD metastasis and 286 non-metastasis samples. The clinical characteristics of the 384 TCGA patients are shown in Table 1 (Additional file 1). No ethical issues were involved, because the sequencing data were obtained by using TCGA database. The edgeR package in Bioconductor was used to screen the DEGs, DEMs and DELs in LUAD metastasis and non-metastasis samples. The genes, miRNAs and lncRNAs were considered as DEGs, DEMs and DELs if P-value $<0.05$ (including up-regulation and down-regulation), respectively (Additional file 2).

\section{Intersection IncRNAs and mRNAs}

Predicted DEMs targets in this study were determined using miRBase targets (http://mirdb.org/miRDB/) to predict target genes, and using miRanda (http://www.micro rna.org/microrna/home.do) to find the lncRNA-miRNA interactions. Then we combined the information of miRNAs predicted and differentially expressed data of TCGA

\section{Table 1 Clinical characteristics of TCGA cohort}

All patients $(n=372)$

\begin{tabular}{ll}
\hline Age at diagnosis (years) & \\
Mean & 65 \\
Range & $38-85$ \\
Sex & \\
Male & $183(49.2 \%)$ \\
Female & $189(50.8 \%)$ \\
Clinical stage & \\
I & $3(0.8 \%)$ \\
Ia & $85(22.9 \%)$ \\
Ib & $101(27.2 \%)$ \\
Ila & $34(9.1 \%)$ \\
Ilb & $53(14.2 \%)$ \\
Illa & $55(14.8 \%)$ \\
Illb & $10(2.7 \%)$ \\
IV & $25(6.7 \%)$ \\
NA & $6(1.6 \%)$ \\
Metastasis & \\
Yes & $25(6.7 \%)$ \\
No & $347(93.3 \%)$ \\
Overall survival & \\
Deaths & \\
Alive & $148(39.8 \%)$ \\
Follow-up (months) & $224(60.2 \%)$ \\
Mean & \\
Range & 30.8 \\
\hline
\end{tabular}


by using Excel to obtain the repeating part to choose the intersection lncRNAs and mRNAs.

\section{GO and KEGG pathway enrichment analysis}

Differentially expressed intersection mRNAs were obtained from the Database for Annotation, Visualization and Integrated Discovery (DAVID), which provided a comprehensive set of functional annotation tools for investigators to understand the biological meaning behind large lists of genes (http://david.abcc.ncifcrf.gov/). Up and down-regulated genes were analyzed, respectively. Two-side Fisher's exact test and $\chi^{2}$ test were used to classify the GO category, and the false discovery rate (FDR) was calculated to correct the P-value,the smaller the FDR, the smaller the error in judging the p-value [17]. The FDR was defined as $F D R=1-\frac{N_{k}}{T}$, where $N_{k}$ refers to the number of Fisher's test P-values less than $\chi^{2}$ test P-values. We computed P-values for the GOs of all the differential genes. Enrichment provides a measure of the significance of the function: as the enrichment increases, the corresponding function can be more specific, which helps us to find those GOs with more concrete function description in the experiment. Within the significant category, the enrichment $\operatorname{Re}$ was given by: $\operatorname{Re}=\left(n_{f} / n\right) /\left(N_{f} / N\right)$ where " $n$ " is the number of flagged genes within the particular category, " $n$ " is the total number of genes within the same category, " $N_{f}$ " is the number of flagged genes in the entire microarray, and " $N$ " is the total number of genes in the microarray [18].

KEGG (http://www.kegg.jp/) was used to analyze the potential functions of these genes participated in the pathways [19]. Still, we turn to the Fisher's exact test and $X^{2}$ test to select the significant pathway, and the threshold of significance was defined by P-value and FDR. The enrichment $\operatorname{Re}$ was calculated like the equation above [20-22]. $\mathrm{P}<0.05$ was considered as the threshold criterion.

\section{Co-expression analysis}

Differentially expressed intersection mRNAs which had similar expression profiles were included in the differentially co-expressed genes analysis to investigate the potential metastatic mechanism of LUAD. The differentially co-expressed genes in LUAD metastasis samples compared to non-metastasis samples were identified via the DCGL package in R. In the network analysis, a degree is the most important parameter of the centrality of a gene within a network that determines the relative importance. Up-regulated and down-regulated mRNAs were showed in pies with different colors. Then, the differential co-expression network was constructed based on the Cytoscape software.

\section{Survival analysis}

The univariable Cox regression analysis was performed to assess the relationship between mRNAs expression levels and LUAD patients overall survival (OS) and recurrence free survival (RFS) time and identify survival-associated genes. The survival curves were plotted and tested using the Kaplan-Meier method and log-rank test. According to the median risk score, LUAD patients were divided into high- and low-risk groups. The mRNAs with logrank $\mathrm{P}<0.05$ between high-risk and low-risk groups were considered statistically significant. $\mathrm{R}$ software and Bioconductor were used for all these analyses. Next, we get target genes by combining significant survival-associated genes and genes incorporated into the gene co-expression network.

\section{Receiver operating characteristic (ROC) curve analysis}

In order to assess the diagnostic value of target genes in LUAD, receiver operating characteristic (ROC) analysis were performed using pROC package in $\mathrm{R}$ language [23]. Diagnostic ability of the prediction model was evaluated, by calculating the area under a ROC curve. The ROC curve was used for classifier evaluation, and was drawn by plotting sensitivity against the false-positive rate. The area under the curve (AUC) under binomial exact confidence interval was calculated to generate the ROC curve.

\section{MiRNA-mRNA-IncRNA network}

According to the relationship among mRNA, miRNA and IncRNA, the posttranscriptional regulation of mRNA transcripts bound by single-stranded miRNAs is basically established. We have established a theory based on IncRNA regulating miRNA abundance by isolating and binding them, acting as a so-called miRNA sponge [24]. Then, according to the theory of ceRNA, we chose the miRNA negatively regulated expression of lncRNAs and mRNAs to construct the miRNA-mRNA-lncRNA network. The miRNA-mRNA-lncRNA network was constructed and visualized using Cytoscape v3.0 [25].

\section{Statistical analysis}

All data in the present study were analyzed using GraphPad Prism 6.0 software. Mean \pm standard deviation and independent-samples t-test was used in the statistical analysis. A P-value less than 0.05 was considered statistically significant.

\section{Results \\ Workflow}

The present study consists of several processes sequentially (Fig. 1), that is TCGA-based RNA-seq data aggregation, and multiple bioinformatics analyses. 


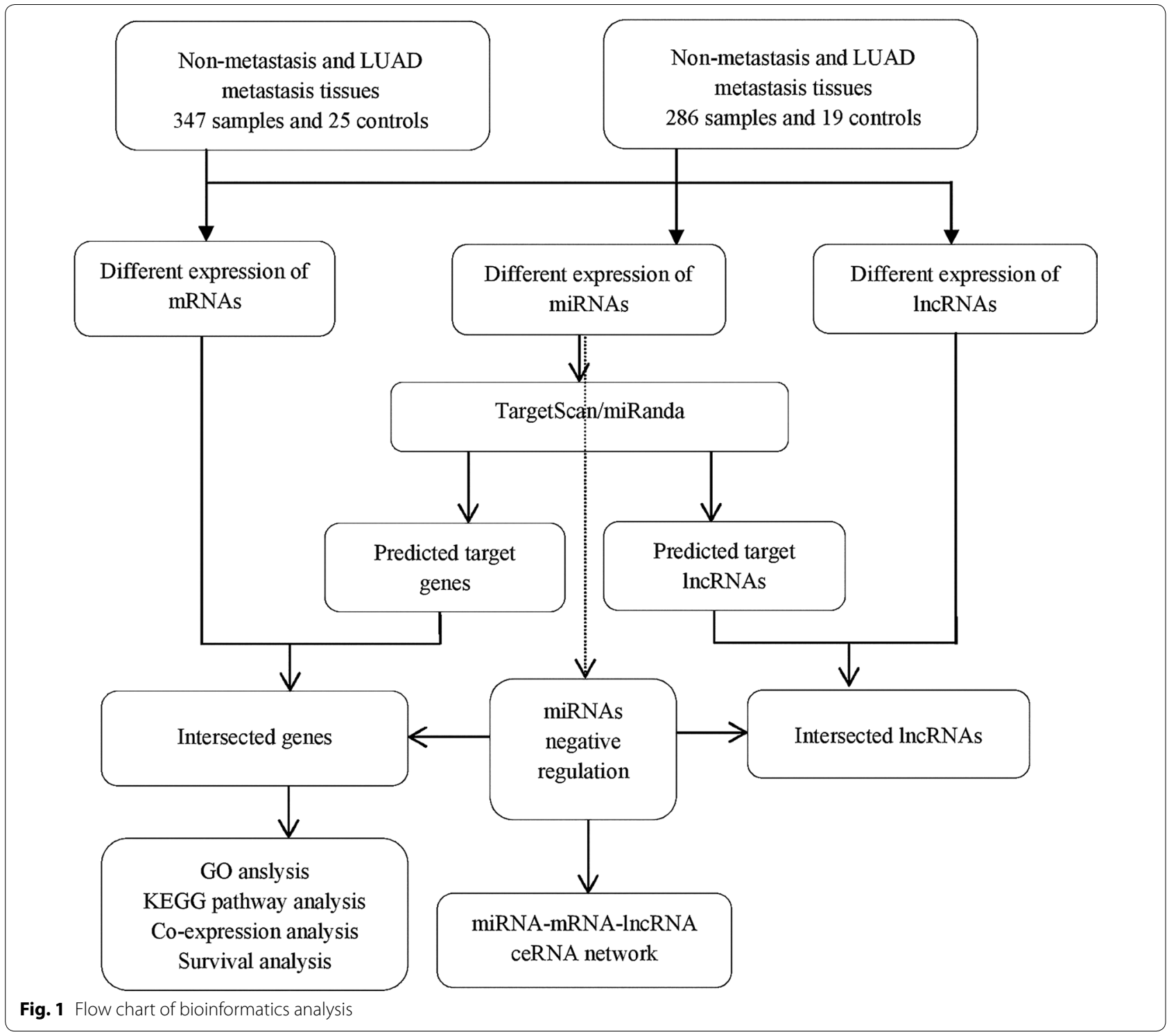

DEGs, DEMs and DELs in LUAD metastasis and non-metastasis samples based on TCGA data

A total of 1019 genes were considered as DEGs in LUAD metastasis samples compared to non-metastasis samples, including 581 (57.0\%) up-regulated and 438 (43.0\%) down-regulated genes. And the top 20 DEGs in LUAD metastasis samples compared with non-metastasis samples were shown in Table 2. 54 miRNAs were considered as DEMs, including 12 (22.2\%) up-regulated and 42 (77.8\%) down-regulated miRNAs. 21 lncRNA s were considered as DELs, including 8 (38.1\%) up-regulated and 13 (61.9\%) down-regulated lncRNAs (Fig. 2).

\section{Intersection IncRNAs and mRNAs}

The mRBase targets method was used to analyse the target mRNAs of DEMs and obtained the 915 miRNA targeted mRNAs. The miRanda method was used to analyse the target lncRNAs of DEM and obtained the 22 miRNAtargeted lncRNAs. Then the study combined the information of miRNAs predicted and differentially expressed data of TCGA, and obtain 915 intersection mRNAs and 20 intersection lncRNAs.

\section{GO and KEGG analysis}

Predicted functions of DEGs in this study were determined by intersection mRNAs. The 915 intersection 
Table 2 The top 20 DEGs in LUAD metastasis samples compared with non-metastasis samples

\begin{tabular}{|c|c|c|c|c|c|}
\hline Gene name & P-value & $\begin{array}{l}\text { Geom mean of intensities in LUAD } \\
\text { non-metastasis }\end{array}$ & $\begin{array}{l}\text { Geom mean of intensities } \\
\text { in LUAD metastasis }\end{array}$ & $\log 2(F C)$ & Style \\
\hline ADRA2A & $2.77 \mathrm{E}-03$ & 138.17 & 57.40 & 1.27 & Up \\
\hline SFTPB & $3.64 \mathrm{E}-02$ & $57,876.78$ & $24,815.46$ & 1.22 & Up \\
\hline IGJ & $1.94 \mathrm{E}-03$ & 6335.86 & 2744.20 & 1.21 & Up \\
\hline CD79A & $2.31 \mathrm{E}-03$ & 365.56 & 160.15 & 1.19 & Up \\
\hline CPZ & $2.26 \mathrm{E}-05$ & 264.00 & 119.83 & 1.14 & Up \\
\hline $\mathrm{CH} 25 \mathrm{H}$ & 4.05E-03 & 140.60 & 68.34 & 1.04 & Up \\
\hline LRRC15 & $1.60 \mathrm{E}-02$ & 271.70 & 133.16 & 1.03 & Up \\
\hline UCHL1 & $4.55 \mathrm{E}-02$ & 380.51 & 784.12 & -1.03 & Down \\
\hline SFRP2 & $1.63 \mathrm{E}-02$ & 1526.84 & 757.42 & 1.01 & Up \\
\hline SKAP1 & $1.25 \mathrm{E}-03$ & 138.21 & 69.33 & 0.99 & Up \\
\hline RNASE1 & $2.25 E-03$ & 7830.26 & 3995.57 & 0.97 & Up \\
\hline CILP2 & 9.77E-03 & 146.06 & 74.91 & 0.96 & Up \\
\hline LTB & $1.57 \mathrm{E}-03$ & 264.47 & 139.19 & 0.93 & Up \\
\hline COL10A1 & $1.65 \mathrm{E}-02$ & 682.85 & 363.03 & 0.91 & Up \\
\hline TRIM16L & $9.68 \mathrm{E}-03$ & 184.04 & 342.52 & -0.89 & Down \\
\hline DERL3 & $1.83 \mathrm{E}-03$ & 602.72 & 327.93 & 0.88 & Up \\
\hline CPXM1 & $2.46 \mathrm{E}-03$ & 209.17 & 113.48 & 0.88 & Up \\
\hline APBA2 & $1.29 \mathrm{E}-02$ & 220.67 & 121.83 & 0.86 & Up \\
\hline PODN & 7.01E-04 & 746.37 & 414.51 & 0.85 & Up \\
\hline FMO3 & $1.62 \mathrm{E}-03$ & 165.01 & 92.10 & 0.84 & Up \\
\hline
\end{tabular}

mRNAs were further analyzed by GO analysis. We analyzed the enrichment of these genes. Enrichment provides a measure of the significance of the function, and as the enrichment increases, the corresponding function is more specific, which helped us to identify GO with a more definitive functional description [26]. The results showed that the up-regulated genes were significantly associated with signal transduction, innate immune response, immune response, blood coagulation, and cell adhesion, while the down-regulated genes were mainly involved in mitotic cell cycle, DNA repairment, mitotic prometaphase, $S$ phase of mitotic cell cycle and $M$ phase of mitotic cell cycle (Fig. 3a, b).

Pathway analysis indicated that 87 pathways corresponded to up-regulated transcripts and up-regulated genes were mainly related to cytokine-cytokine receptor interaction, osteoclast differentiation, cell adhesion molecules (CAMs), PI3K-Akt signaling pathway and focal adhesion, while down-regulated DEGs were associated with Fanconi anemia pathway, mismatch repair, spliceosome, DNA replication and cell cycle (Fig. 3c, d).

\section{Co-expression network}

The intersection genes were used to construct a gene coexpression network. According to the node connectivity, genes can be further classified into hub genes. Hub genes are very important nodes and represent a small proportion of nodes with maximal information exchange with other nodes in the gene co-expression network. The entire network consisted of 145 nodes and 658 connections. Solid lines present positive correlation and dotted lines present negative correlation. The networks indicate that one gene is correlated with several genes and vice versa. A higher degree for one gene meant that the gene played a more important role in this network. Among the co-expression interactions network, there are 22 genes in the co-expression network with the degree over 20, including SASH3, WAS, CD53, NCKAP1L, PTPRC, PTPN7, CD4, CYTH4, ARHGAP9, FERMT3, TRAF3IP3, EVI2B, SNX20, LAPTM5, BTK, IKZF1, ARHGAP30, CCR5, IL10RA, IL16, LCP2 and PSTPIP1. These genes imply that they have connections with many other gene nodes (Fig. 4).

\section{Survival analysis}

In the survival analysis, 114 genes were found to be significant in LUAD patients (log-rank test, $\mathrm{P}<0.05)$. While we choose 14 target genes which were obtained by combining significant survival associated genes and genes incorporated into the gene co-expression network. In order to reveal association between the 14 target genes expression levels and LUAD prognosis, we performed Kaplan-Meier survival curves. The results show that patients with lower expression levels of five genes including ARHGAP11A, 

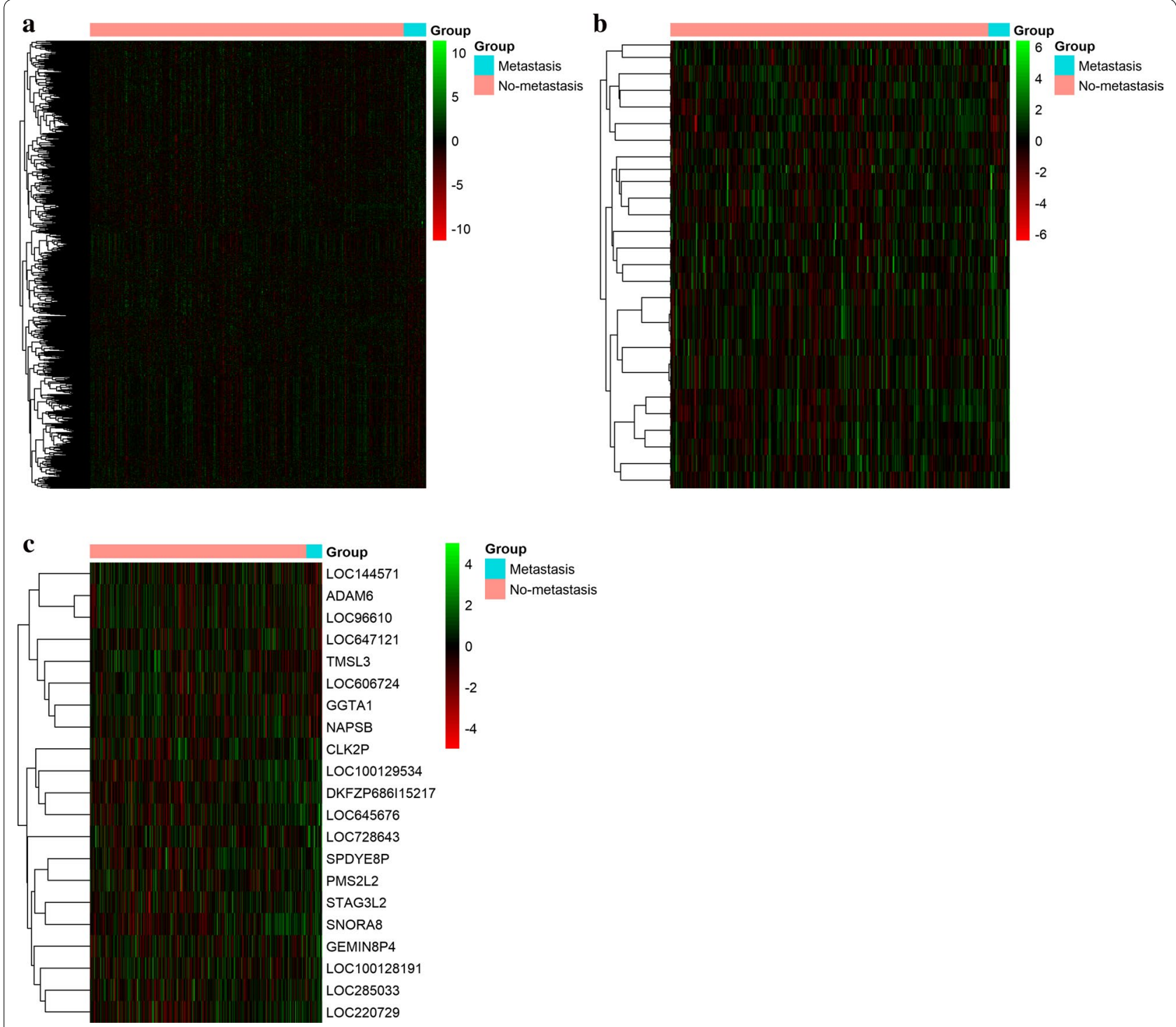

Fig. 2 Heatmap for hierarchical cluster analysis of DEGs, DEMs and DELs expression levels change between LUAD metastasis and non-metastasis tissues $(\mathbf{a}-\mathbf{c})$. Colors ranged from green (low expression) to red (high expression), representing the relative expression levels of DEGs, DEMs and DELs

ASPM, HELLS, PRC1 and TMPO have better survival prognoses than those with higher expression levels of these five genes in LUAD. Patients with higher expression levels of the rest nine genes (ARHGAP30, CD52, IL16, IRF8, P2RY13, PRKCB, PTPRC, SASH3, TRAF3IP3) were associated with poor survival in patients with LUAD (all log-rank $\mathrm{P}<0.05$ ) (Fig. 5).

\section{ROC curve analysis}

In order to assess the discriminatory ability of the 14 target genes among LUAD metastasis and non-metastasis samples generated from TCGA database, ROC curve analyses were conducted and AUC were calculated.
As Fig. 6 shown, the AUC of 11 target genes (ARHGAP11A, ARHGAP30, ASPM, IL16, IRF8, P2RY13, PRC1, PRKCB, PTPRC, SASH3, and TRAF3IP3) were more than 0.6. The AUC of CD52, HELLS and TMPO was respective $0.578,0.57$ and 0.577 , less than 0.6 . IL16 had the largest AUC in those 14 target genes.

\section{MiRNA-mRNA-IncRNA network}

Besides the 14 target genes which were obtained by combining significant survival associated genes and genes incorporated into the gene co-expression network, we also performed miRNA-mRNA-lncRNA to identify lncRNAs which acted as ceRNAs in LUAD 


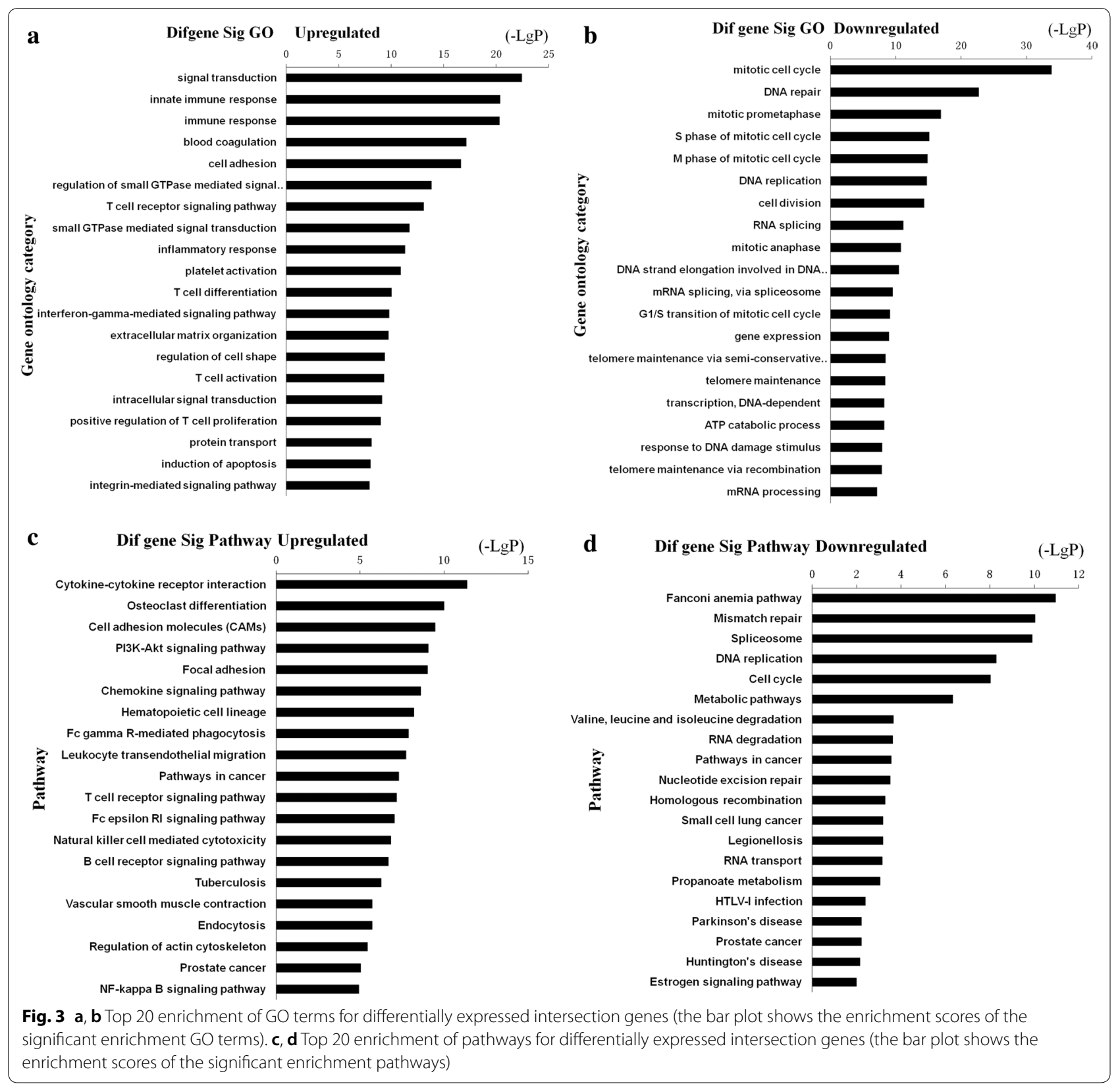

pathogenesis. The miRNA-mRNA-lncRNA relationship was integrated into the ceRNA network through negative regulation. Based on the interaction network of miRNA-mRNA, miRNA-lncRNA and lncRNA-mRNA, we obtained 37 feed-forward loop networks and constructed general miRNA-lncRNA-mRNA feed-forward loop network (data not shown). In our study, the results identified that 2 lncRNAs (LOC96610 and ADAM6), 22 miRNAs and 4 mRNAs (LAX1, DERL3, MEI1 and CPNE5) were involved in the ceRNA network. The functions of LOC96610 and ADAM6 acting as ceRNAs were predicted through pathway analysis of 4 mRNAs (LAX1, DERL3, MEI1 and CPNE5) in the miRNA-IncRNAmRNA interaction network. The results indicated that four mRNAs participated in three upregulated pathways which involved in immune response, endoplasmic reticulum unfolded protein response and male meiosis I. As a consequence, it was believed that compared with the other lncRNAs, these two IncRNAs (LOC96610 and ADAM6) might played more significant functions in the whole ceRNA network (Fig. 7). 


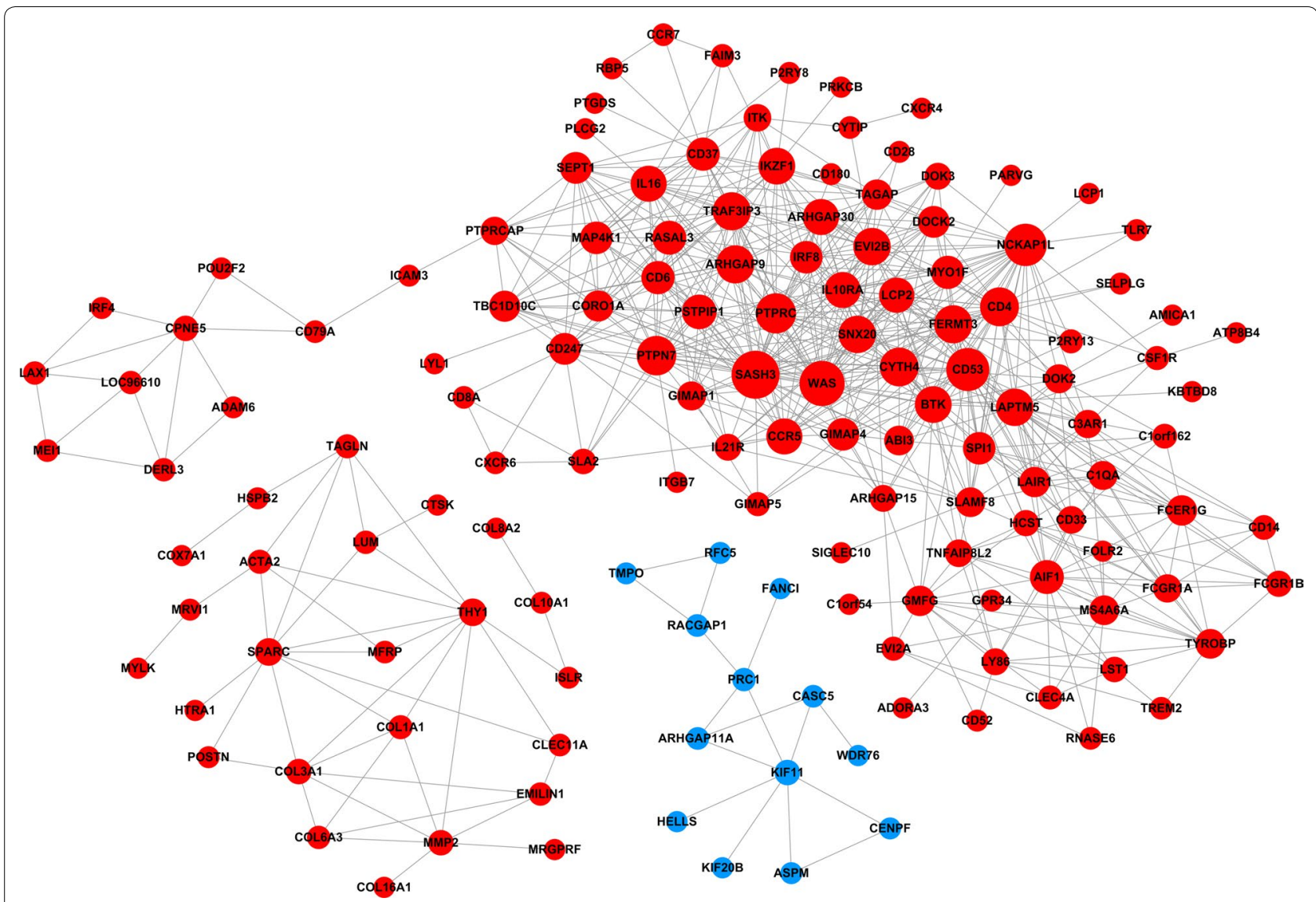

Fig. 4 The co-expression network constructed by Cytoscape software. Proteins are represented with color nodes, and interactions are represented with edges. Red color indicated up-regulation; blue color indicated down-regulation

\section{Discussion}

Even though there was a new development of immunotherapy and targeted therapy of tumors, once the systematic metastasis of cancer cell occurred, the 5-year survival rate would decrease at less by $10 \%$ [27], and the survival of LUAD is far from satisfactory. Early diagnosis plays a critical role in the prevention and treatment of cancer, including LUAD. And researchers are continuously seeking for new biomarkers or targets for LUAD prevention, diagnose and treatment.

RNA-seq data and microarray-based expression profiling data provide a more comprehensive and accurate understanding of carcinogenesis and cancer progression at the molecular level. In our study, we analyzed the differences in the mRNAs, miRNAs, IncRNAs expression according to raw sequencing data of LUAD metastasis and non-metastasis samples from TCGA. We found that 1019 DEGs, 54 DEMs and 21 DELs are differently expressed in LUAD metastasis samples compared to non-metastasis samples. Combined with DEMs targeted mRNAs, we obtained 915 intersection mRNAs which were used to conduct GO and KEGG pathway analysis. According to the results of GO enrichment, the most significantly up-regulated genes were associated with signal transduction, while the most significantly downregulated genes were mainly involved in mitotic cell cycle. KEGG pathway analysis showed that pathways corresponded to up-regulated transcripts were mainly related to cytokine-cytokine receptor interaction, pathways corresponded to down-regulated transcripts were mainly related to fanconi anemia pathway.

Generally, the common influence of interaction in interacting genes could not only decrease the complexity of biological network, but also benefit to explore 


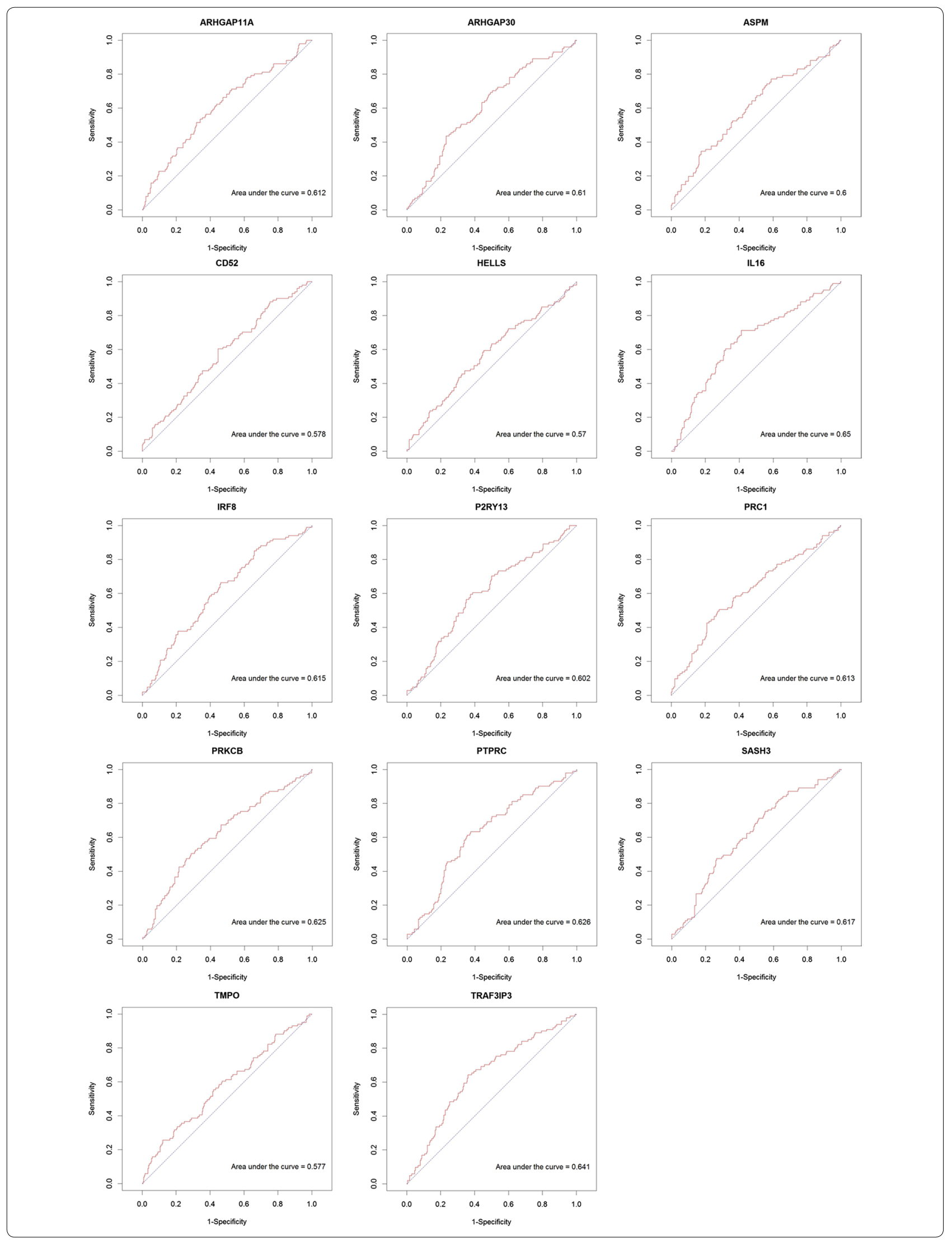



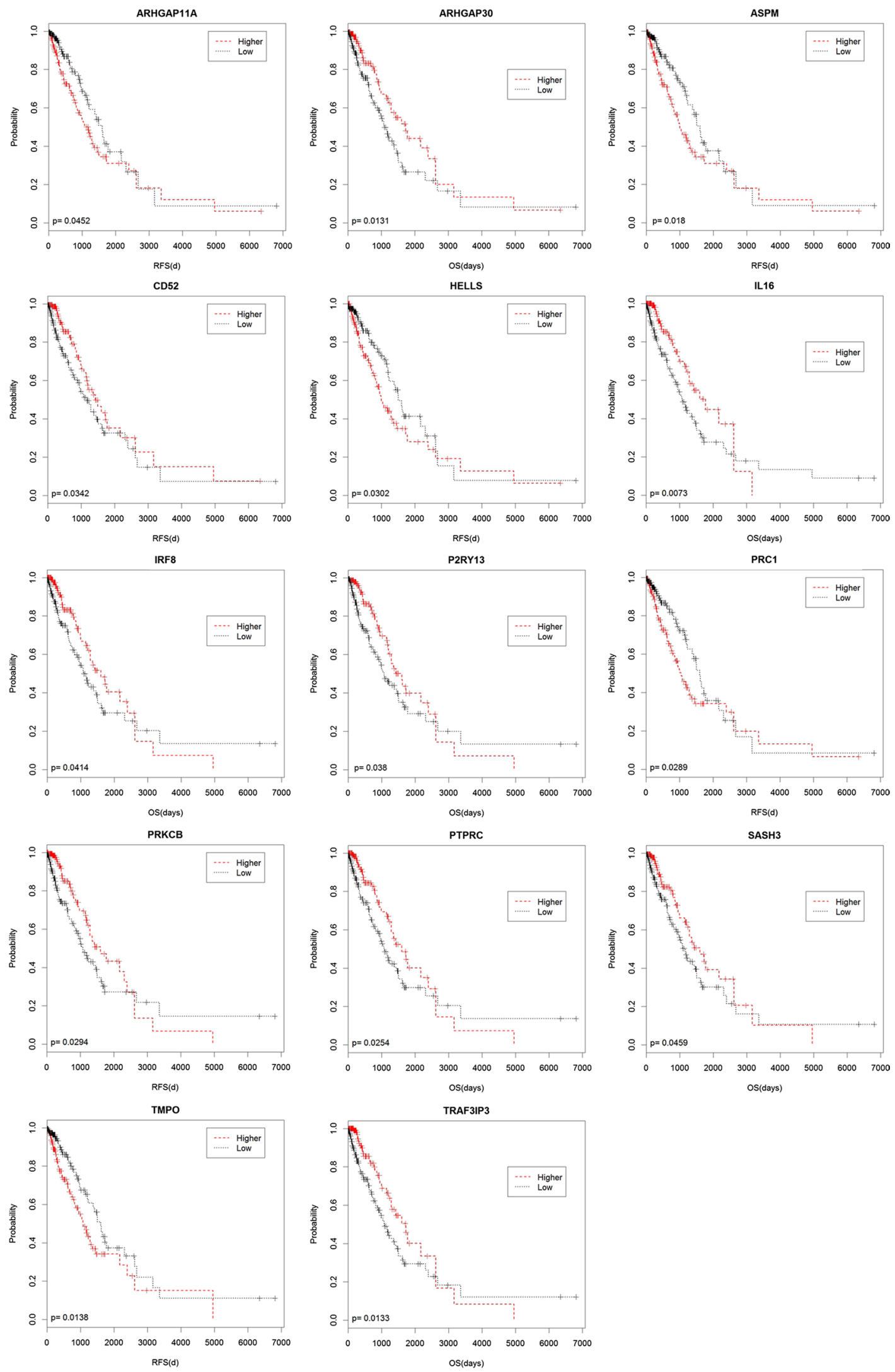
Fig. 6 The receiver operating characteristic (ROC) curves for 14 target genes. The area under the curve (AUC) under binomial exact confidence interval was calculated to generate the ROC curve

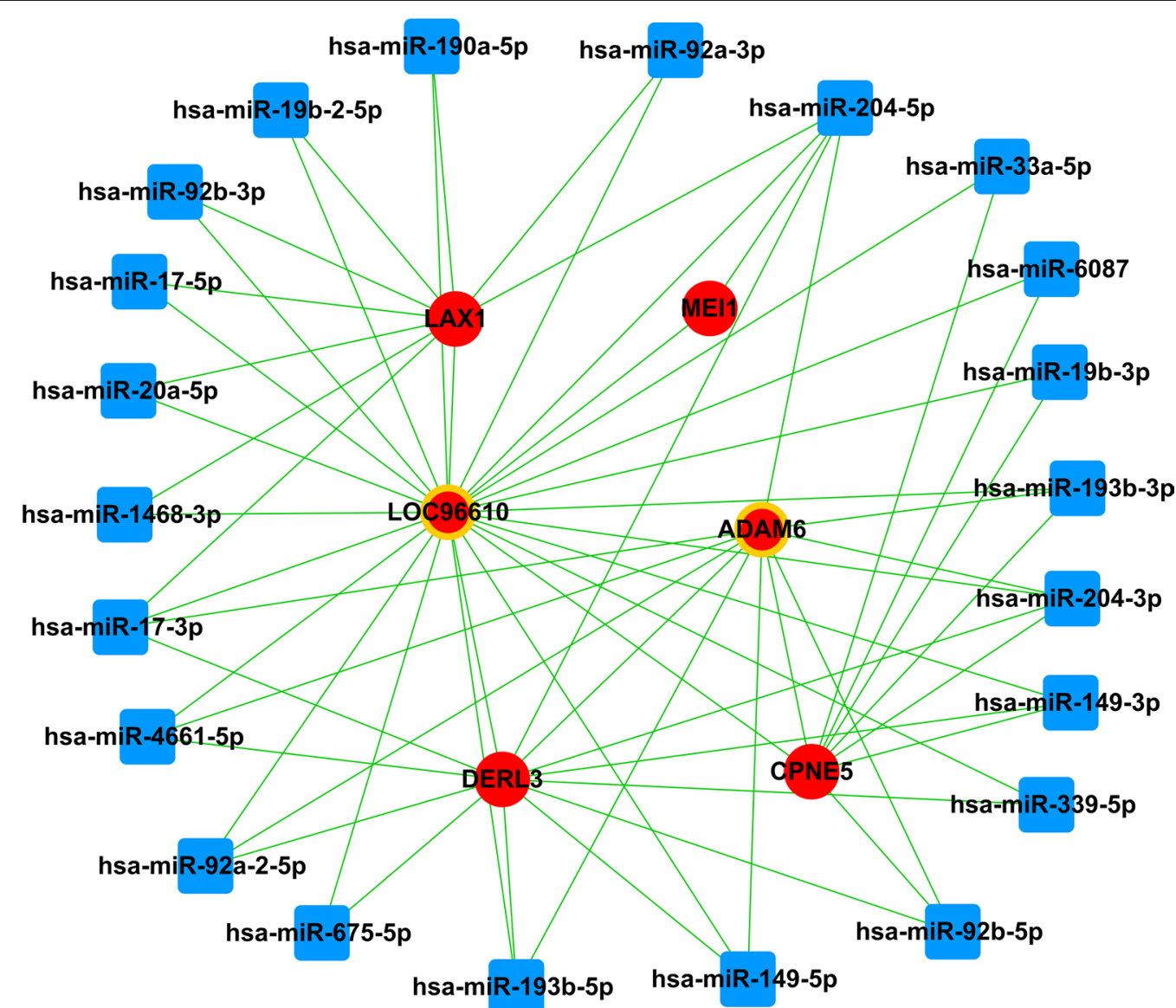

Fig. 7 The miRNA-mRNA-IncRNA ceRNA network. Red balls represent up-regulated mRNAs, blue diamonds, down-regulated miRNAs, and red balls surrounded by yellow rings, up-regulated IncRNAs

meaningful biological information for the researchers, providing further scientific basis for therapy and study of disease [28]. In our study, the co-expression gene network based on the intersection genes provided an insight of correlation between genes. Here, we found that some genes, such as SASH3, WAS and CD53, had connections with many other gene nodes in LUAD. They might be considered as important biomarkers which account for the metastasis mechanism of LUAD. In addition, survival analysis determined that 114 genes increased or decreased expression is significantly associated with LUAD patients shorter OS or RFS, suggesting that these genes may be valuable predictive factors for LUAD patient's survival. Finally, 14 target genes were obtained by combining significant survival associated genes and were incorporated into the gene co-expression network.
In the target genes, five genes (ARHGAP30, IL16, PTPRC, SASH3 and TRAF3IP3) were with the degree over 20, which implied the five genes not only had better important regulatory value for the network, but also had diagnostic value of LUAD metastasis. ARHGAP30, a previously uncharacterized RhoGAP domain-containing protein, as a candidate Wrch1-binding protein. ARHGAP30 is closely related to the Cdc42-specific RhoGAP CdGAP [29], and together they form a subgroup of the RhoGAP proteins. Wang et al. [30] identified ARHGAP30 served as a key regulator for p53 acetylation, and suggested ArhGAP30 as both prognostic marker and potential therapeutic target for colorectal cancer. Besides, ARHGAP30 was found had significantly improved OS of pancreatic ductal adenocarcinoma [31]. Interleukin-16 (IL-16) is 
a pro-inflammatory cytokine [32] and chemo attractant for a broad variety of immune cell types with CD4 co-receptors [33]. Serum IL-16 levels have been associated with other cancers, such as multiple myeloma [34], gastric cancer [35], and colorectal cancer. Besides, IL-16 was reported to may act as a key mediator in pre metastatic niches that drives the establishment of lung metastasis and may represent a suitable therapeutic target [36]. PTPRC, also known as CD45, encodes a member of the protein tyrosine phosphatase (PTP) family, which comprises proteins commonly activated in tumors [37]. A previous report suggested that up-regulation of PTPRC resulted in high levels of inflammatory cytokines [38]. It is reported that PTPRC was predicted to interact with CXCR4, and PTPRC might also play a role in colon cancer metastasis [39]. SASH3 encodes a signaling adapter protein, containing a SLY motif in the $\mathrm{N}$-terminal region, a SH3 motif and a SAM motif in the C-terminal region [40]. SAM families of receptors are known to play a role in many developmental processes including cell migration, neuronal formation and angiogenesis [40]. Schieffer et al. [41] revealed SASH3 as a hub gene was highly correlated with diverticulitis patients compared to non-diverticulosis controls. TRAF3IP3, also known as TRAF3-interacting JNK Activating Modulator (T3JAM), was originally identified as a protein that interacts specifically with Tumor necrosis factor receptor-associated factor 3 (TRAF3) to activate JNK in human kidney cells [42]. TRAF3IP3 is expressed in the immune system and participates in cell maturation, tissue development, and immune response. Nasarre et al. [43] identified new functions of TRAF3IP3 in melanoma and angiogenesis, emphasizing its physiological relevance as a potential target for cancer therapy.

Moreover, our study identified that $2 \operatorname{lncRNAs}$ (LOC96610 and ADAM6), 22 miRNAs and 4 mRNAs (LAX1, DERL3, MEI1 and CPNE5) were involved in the miRNA-lncRNA-mRNA interaction network. LOC96610, located at 22q11.22, the official symbol is BMS1P20 (BMS1, ribosome biogenesis factor pseudogene 20). As a survival-related lncRNA, BMS1P20 was found significantly correlated with the pathogenesis, development and metastasis of liver hepatocellular carcinoma [44]. Furthermore, Sui et al. [45] reported that BMS1P20 positively correlated with overall survival of LUAD. ADAM6 (ADAM metallopeptidase domain 6), located at $14 \mathrm{q} 32.33$. ADAM is a family of membrane proteins involved in cell-cell adhesion and cell-matrix adhesion. It is characterized by a disintegrin and metalloprotease domain with an epidermal growth factorlike region and harbors both adhesion and proteolytic domains implicated in integrin function and matrix degradation [46, 47]. It is reported that an exploratory biomarker panel derived from ADAM6 conferred prognostic utility for melanoma recurrence and death [48].

\section{Conclusions}

In summary, our findings documented that 1019 DEGs, 54 DEMs and 21 DELs were differently expressed in LUAD metastatic samples compared with non-metastatic samples. Among these altered mRNAs, many are significantly associated with LUAD patient's survival time, and might play critical roles in LUAD metastasis. Our study highlights the important roles of mRNAs, miRNAs and lncRNAs in LUAD metastasis and may provide useful candidates as diagnostic markers and potential targets for LUAD therapy. The present study also has a few limitations, for example, the data used were obtained from TCGA, rather than directly from LUAD patients, thus a series of verification experiments must be performed to confirm our results. Overall, our findings will improve our understanding of the molecular mechanisms of LUAD and aid in finding potential targets for diagnostic and therapeutic usage.

\section{Additional files}

Additional file 1. Clinical data of LUAD from TCGA cohort.

Additional file 2. DEGs in LUAD metastasis samples compared with nonmetastasis samples.

\section{Abbreviations}

LUAD: lung adenocarcinoma; DEGs: differentially expressed genes; DEMs: differentially expressed miRNAs; DELs: differentially expressed IncRNAs; GO: gene ontology; KEGG: Kyoto Encyclopedia of Genes and Genomes; TCGA: The Cancer Genome Atlas; FDR: false discovery rate; ROC: receiver operating characteristic; DAVID: Database for Annotation, Visualization and Integrated Discovery; AUC: area under the curve; OS: overall survival; RFS: recurrence free survival; CAMs: cell adhesion molecules.

\section{Authors' contributions}

ZJ and QDC designed and edited this study; LLF, PML and XWH searched the databases and collected full-text papers; FZR, WT and LYY extracted and analysed the data; ZYK and LWP wrote the manuscript. All authors read and approved the final manuscript.

\section{Author details \\ ${ }^{1}$ Department of Pharmacy, The First Affiliated Hospital of Zhengzhou Univer- sity, Zhengzhou 450052, Henan, China. ${ }^{2}$ Department of Clinical Laboratory, The Third People's Hospital of Henan Province, Zhengzhou 450052, Henan, China. ${ }^{3}$ Cancer Center, The First Affiliated Hospital of Zhengzhou University, Zhengzhou 450052, Henan, China. ${ }^{4}$ Department of Clinical Laboratory, The First Affiliated Hospital of Zhengzhou University, Key Laboratory of Laboratory Medicine of Henan Province, Zhengzhou 450052, Henan, China. ${ }^{5}$ National Engineering Laboratory for Internet Medical Systems and Applications, Zheng-} zhou 450052, Henan, China.

\section{Acknowledgements}

The authors would like to express our sincere thanks for sharing the data from The Cancer Genome Atlas (TCGA) database. 


\section{Competing interests}

The authors declare that they have no competing interests.

\section{Availability of data and materials}

All data generated or analyzed during this study are included either in this article or in the Additional files.

\section{Consent for publication}

Not applicable.

\section{Ethics approval and consent to participate}

Ethical approval for this investigation was obtained from the Research Ethics Committee of First Affiliated Hospital of Zhengzhou University and with the 1964 Helsinki declaration and its later amendments.

\section{Funding}

This study was supported by grants from the National Natural Science Foundation of China (Grant No. 71673254). The National Key Research and Development Program of China-The construction and promotion of the demonstration system of based on telemedicine/Mhealth network (Grant No. 2017YFC0909900), Special funds from central government for the guidance of local science and technology development: Construction and demonstration of telemedicine big data application system for precision medicine. Special funds of major science and technology project in Henan province (2016): Construction and demonstration application of medical and health big data analysis system based on telemedicine cloud platform (Grant No. 151100310800). Doctor research team fund from the first affiliated hospital of Zhengzhou University for the in-hospital Interdisciplinary Collaboration Research: The impact of air pollution exposure levels on lung cancer and its potential molecular mechanism, based on satellite remote sense big data (Grant No. 2016-BSTDJJ-15).

\section{Publisher's Note}

Springer Nature remains neutral with regard to jurisdictional claims in published maps and institutional affiliations.

\section{Received: 17 July 2018 Accepted: 6 December 2018}

Published online: 27 December 2018

\section{References}

1. Torre LA, Bray F, Siegel RL, Ferlay J, Lortet-Tieulent J, Jemal A. Global cancer statistics, 2012. CA Cancer J Clin. 2015;65:87-108.

2. Siegel RL, Miller KD, Jemal A. Cancer statistics, 2016. CA Cancer J Clin. 2016;66:7-30

3. Li JD, Feng QC, Qi Y, Cui G, Zhao S. PPARGC1A is upregulated and facilitates lung cancer metastasis. Exp Cell Res. 2017;359:356-60.

4. Morgensztern D, Ng SH, Gao F, Govindan R. Trends in stage distribution for patients with non-small cell lung cancer: a National Cancer Database survey. J Thorac Oncol. 2010;5:29-33.

5. Pan W. A comparative review of statistical methods for discovering differentially expressed genes in replicated microarray experiments. Bioinformatics. 2002;18:546-54.

6. Hu Z, Fan C, Oh DS, Marron JS, He X, Qaqish BF, Livasy C, Carey LA, Reynolds E, Dressler $L$, et al. The molecular portraits of breast tumors are conserved across microarray platforms. BMC Genomics. 2006;7:96.

7. Munker R, Calin GA. MicroRNA profiling in cancer. Clin Sci. 2011:121:141-58.

8. Ambros $V$. The functions of animal microRNAs. Nature. 2004;431:350-5.

9. Kong YW, Cannell IG, de Moor CH, Hill K, Garside PG, Hamilton TL, Meijer HA, Dobbyn HC, Stoneley M, Spriggs KA, et al. The mechanism of microRNA-mediated translation repression is determined by the promoter of the target gene. Proc Natl Acad Sci USA. 2008;105:8866-71.

10. Xue J, Niu J, Wu J, Wu ZH. MicroRNAs in cancer therapeutic response: friend and foe. World J Clin Oncol. 2014;5:730-43.

11. Ling $H$, Fabbri $M$, Calin GA. MicroRNAs and other non-coding RNAs as targets for anticancer drug development. Nat Rev Drug Discov. 2013;12:847-65.
12. Calin GA, Croce CM. MicroRNA signatures in human cancers. Nat Rev Cancer. 2006;6:857-66.

13. Gibb EA, Brown CJ, Lam WL. The functional role of long non-coding RNA in human carcinomas. Mol Cancer. 2011;10:38.

14. Gupta RA, Shah N, Wang KC, Kim J, Horlings HM, Wong DJ, Tsai MC, Hung T, Argani P, Rinn JL, et al. Long non-coding RNA HOTAIR reprograms chromatin state to promote cancer metastasis. Nature. 2010;464:1071-6.

15. Trimarchi T, Bilal E, Ntziachristos P, Fabbri G, Dalla-Favera R, Tsirigos A, Aifantis I. Genome-wide mapping and characterization of Notch-regulated long noncoding RNAs in acute leukemia. Cell. 2014;158:593-606.

16. Tan JY, Sirey T, Honti F, Graham B, Piovesan A, Merkenschlager M, Webber C, Ponting CP, Marques AC. Corrigendum: extensive microRNA-mediated crosstalk between IncRNAs and mRNAs in mouse embryonic stem cells. Genome Res. 2015:25(1410):1411.

17. Dupuy D, Bertin N, Hidalgo CA, Venkatesan K, Tu D, Lee D, Rosenberg J, Svrzikapa N, Blanc A, Carnec A, et al. Genome-scale analysis of in vivo spatiotemporal promoter activity in Caenorhabditis elegans. Nat Biotechnol. 2007;25:663-8.

18. Schlitt T, Palin K, Rung J, Dietmann S, Lappe M, Ukkonen E, Brazma A. From gene networks to gene function. Genome Res. 2003;13:2568-76.

19. Zhang T, Jiang M, Chen L, Niu B, Cai Y. Prediction of gene phenotypes based on GO and KEGG pathway enrichment scores. Biomed Res Int. 2013;2013:870795

20. Kanehisa M, Goto S, Kawashima S, Okuno Y, Hattori M. The KEGG resource for deciphering the genome. Nucleic Acids Res. 2004;32:D277-80.

21. Yi M, Horton JD, Cohen JC, Hobbs HH, Stephens RM. WholePathwayScope: a comprehensive pathway-based analysis tool for high-throughput data. BMC Bioinform. 2006;7:30.

22. Draghici S, Khatri P, Tarca AL, Amin K, Done A, Voichita C, Georgescu C, Romero R. A systems biology approach for pathway level analysis. Genome Res. 2007:17:1537-45.

23. Robin X, Turck N, Hainard A, Tiberti N, Lisacek F, Sanchez JC, Muller M. pROC: an open-source package for $\mathrm{R}$ and $\mathrm{S}+$ to analyze and compare ROC curves. BMC Bioinform. 2011:12:77.

24. Li CY, Liang GY, Yao WZ, Sui J, Shen $X$, Zhang YQ, Peng H, Hong WW, Ye YC, Zhang ZY, et al. Integrated analysis of long non-coding RNA competing interactions reveals the potential role in progression of human gastric cancer. Int J Oncol. 2016:48:1965-76.

25. Shannon P, Markiel A, Ozier O, Baliga NS, Wang JT, Ramage D, Amin $\mathrm{N}$, Schwikowski B, Ideker T. Cytoscape: a software environment for integrated models of biomolecular interaction networks. Genome Res. 2003:13:2498-504.

26. Li X, Chen H, Li J, Zhang Z. Gene function prediction with gene interaction networks: a context graph kernel approach. IEEE Trans Inf Technol Biomed. 2010;14:119-28.

27. Siegel RL, Miller KD, Jemal A. Cancer statistics, 2015. CA Cancer J Clin. 2015:65:5-29.

28. Chen K, Li Y, Xu H, Zhang C, Li Z, Wang W, Wang B. An analysis of the gene interaction networks identifying the role of PARP1 in metastasis of nonsmall cell lung cancer. Oncotarget. 2017;8:87263-75.

29. Lamarche-Vane N, Hall A. CdGAP, a novel proline-rich GTPase-activating protein for Cdc42 and Rac. J Biol Chem. 1998;273:29172-7.

30. Wang J, Qian J, Hu Y, Kong X, Chen H, Shi Q, Jiang L, Wu C, Zou W, Chen $Y$, et al. ArhGAP30 promotes p53 acetylation and function in colorectal cancer. Nat Commun. 2014:5:4735

31. Liao X, Huang K, Huang R, Liu X, Han C, Yu L, Yu T, Yang C, Wang X, Peng T. Genome-scale analysis to identify prognostic markers in patients with early-stage pancreatic ductal adenocarcinoma after pancreaticoduodenectomy. OncoTargets Ther. 2017;10:4493-506.

32. Glass WG, Sarisky RT, Vecchio AM. Not-so-sweet sixteen: the role of IL-16 in infectious and immune-mediated inflammatory diseases. J Interferon Cytokine Res. 2006:26:511-20.

33. CruikshankWW, Kornfeld H, Center DM. Interleukin-16. J Leukoc Biol. 2000;67:757-66.

34. Alexandrakis MG, Passam FH, Kyriakou DS, Christophoridou AV, Perisinakis K, Hatzivasili A, Foudoulakis A, Castanas E. Serum level of interleukin-16 in multiple myeloma patients and its relationship to disease activity. Am J Hematol. 2004;75:101-6.

35. Gao LB, Rao L, Wang YY, Liang WB, Li C, Xue H, Zhou B, Sun H, Li Y, LV $M L$, et al. The association of interleukin-16 polymorphisms with IL-16 
serum levels and risk of colorectal and gastric cancer. Carcinogenesis. 2009;30:295-9.

36. Donati K, Sepult C, Rocks N, Blacher S, Gerard C, Noel A, Cataldo D. Neutrophil-derived Interleukin 16 in premetastatic lungs promotes breast tumor cell seeding. Cancer Growth Metastasis. 2017:10:1179064417738513.

37. Hendriks WJ, Pulido R. Protein tyrosine phosphatase variants in human hereditary disorders and disease susceptibilities. Biochim Biophys Acta. 2013;1832:1673-96.

38. Ostman A, Hellberg C, Bohmer FD. Protein-tyrosine phosphatases and cancer. Nat Rev Cancer. 2006;6:307-20

39. Chu S, Wang H, Yu M. A putative molecular network associated with colon cancer metastasis constructed from microarray data. World J Surg Oncol. 2017;15:115.

40. Beer S, Simins AB, Schuster A, Holzmann B. Molecular cloning and characterization of a novel SH3 protein (SLY) preferentially expressed in lymphoid cells. Biochim Biophys Acta. 2001;1520:89-93.

41. Schieffer KM, Choi CS, Emrich S, Harris L, Deiling S, Karamchandani DM, Salzberg A, Kawasawa YI, Yochum GS, Koltun WA. RNA-seq implicates deregulation of the immune system in the pathogenesis of diverticulitis. Am J Physiol Gastrointest Liver Physiol. 2017;313:G277-84.

42. Dadgostar H, Doyle SE, Shahangian A, Garcia DE, Cheng G. T3JAM, a novel protein that specifically interacts with TRAF3 and promotes the activation of JNK(1). FEBS Lett. 2003;553:403-7.
43. Nasarre P, Bonilla IV, Metcalf JS, Hilliard EG, Klauber-DeMore N. TRAF3interacting protein 3 , a new oncotarget, promotes tumor growth in melanoma. Melanoma Res. 2018;28:185-94.

44. Xu C, Ping Y, Zhao H, Ning S, Xia P, Wang W, Wan L, Li J, Zhang L, Yu L, Xiao Y. LncNetP, a systematical IncRNA prioritization approach based on ceRNA and disease phenotype association assumptions. Oncotarget. 2017:8:114603-12.

45. Sui J, Li YH, Zhang YQ, Li CY, Shen X, Yao WZ, Peng H, Hong WW, Yin LH, Pu YP, Liang GY. Integrated analysis of long non-coding RNA associated ceRNA network reveals potential IncRNA biomarkers in human lung adenocarcinoma. Int J Oncol. 2016;49:2023-36.

46. Wolfsberg TG, Primakoff P, Myles DG, White JM. ADAM, a novel family of membrane proteins containing A disintegrin And metalloprotease domain: multipotential functions in cell-cell and cell-matrix interactions. J Cell Biol. 1995;131:275-8.

47. Perry AC, Jones R, Hall L. Analysis of transcripts encoding novel members of the mammalian metalloprotease-like, disintegrin-like, cysteine-rich (MDC) protein family and their expression in reproductive and nonreproductive monkey tissues. Biochem J. 1995;312(Pt 1):239-44.

48. Chiu CG, Nakamura Y, Chong KK, Huang SK, Kawas NP, Triche T, Elashoff D, Kiyohara E, Irie RF, Morton DL, Hoon DS. Genome-wide characterization of circulating tumor cells identifies novel prognostic genomic alterations in systemic melanoma metastasis. Clin Chem. 2014;60:873-85.
Ready to submit your research? Choose BMC and benefit from:

- fast, convenient online submission

- thorough peer review by experienced researchers in your field

- rapid publication on acceptance

- support for research data, including large and complex data types

- gold Open Access which fosters wider collaboration and increased citations

- maximum visibility for your research: over 100M website views per year

At BMC, research is always in progress.

Learn more biomedcentral.com/submissions 